\title{
Cavity-Enhanced Raman Spectroscopy in the Biosciences: In Situ, Multicomponent, and Isotope Selective Gas Measurements To Study Hydrogen Production and Consumption by Escherichia coli
}

\author{
Thomas W. Smith and Michael Hippler*i) \\ Department of Chemistry, University of Sheffield, Sheffield S3 7HF, United Kingdom
}

Supporting Information

\begin{abstract}
Recently we introduced cavity-enhanced Raman spectroscopy (CERS) with optical feedback cw-diode lasers as a sensitive analytical tool. Here we report improvements made on the technique and its first application in the biosciences for in situ, multicomponent, and isotope selective gas measurements to study hydrogen production and consumption by Escherichia coli. Under anaerobic conditions, cultures grown on rich media supplemented with D-glucose or glycerol produce $\mathrm{H}_{2}$ and simultaneously consume some of it. By introducing $\mathrm{D}_{2}$ in the headspace, hydrogen production and consumption could be separated due to the distinct spectroscopic signatures of isotopomers. Different phases with distinctly different kinetic regimes of $\mathrm{H}_{2}$ and $\mathrm{CO}_{2}$ production and $\mathrm{D}_{2}$ consumption were identified. Some of the $\mathrm{D}_{2}$ consumed is converted back to $\mathrm{H}_{2}$ via $\mathrm{H} / \mathrm{D}$ exchange with the solvent. HD was formed only as a minor component. This reflects either that $\mathrm{H} / \mathrm{D}$ exchange at hydrogenase active sites is rapid compared to the rate of recombination, rapid recapture of HD occurs after the molecule is formed, or that the active sites where $\mathrm{D}_{2}$ oxidation and proton reduction occur are physically separated. Whereas in glucose supplemented cultures, addition of $\mathrm{D}_{2}$ led to an increase in $\mathrm{H}_{2}$ produced, while the yield of $\mathrm{CO}_{2}$ remained unchanged; with glycerol, addition of $\mathrm{D}_{2}$ led not only to increased yields of $\mathrm{H}_{2}$, but also significantly increased $\mathrm{CO}_{2}$ production, reflecting an impact on fermentation pathways. Addition of $\mathrm{CO}$ was found to completely inhibit $\mathrm{H}_{2}$ production and significantly reduce $\mathrm{D}_{2}$ oxidation, indicating at least some role for $\mathrm{O}_{2}$-tolerant Hyd-1 in $\mathrm{D}_{2}$ consumption.
\end{abstract}

$\mathrm{W}$ ith concerns about greenhouse gas emissions and diminishing supplies of fossil fuels, focus is turning to renewable, net carbon-neutral sources of energy. Among these, dihydrogen $\left(\mathrm{H}_{2}\right)$ holds promise as a possible alternative, although there still remain challenges that must be overcome before a large-scale "Hydrogen Economy" could be feasible, including efficient storage, distribution, and improvements in sustainable production. ${ }^{1-4}$ Biologically derived "biohydrogen" is a promising alternative to abiotic $\mathrm{H}_{2}$ production. $^{5-7}$ Many microorganisms can produce $\mathrm{H}_{2}$ either from breakdown of organic substrates or via light-driven processes. ${ }^{8,9}$ The vast majority of microbial $\mathrm{H}_{2}$ is generated by hydrogenases (see ref 10 for a recent review). Despite utilizing comparatively "poor", non-noble metals, hydrogenases achieve very high activities while operating under the relatively mild conditions of the intracellular environment. Unfortunately, most hydrogenases are sensitive to $\mathrm{O}_{2} \cdot{ }^{7,10}$ Any industrial scale biohydrogen reactor would therefore require systems to monitor levels of $\mathrm{O}_{2}$, to ensure efficient $\mathrm{H}_{2}$ production and for safe operation. Simultaneous measurements of $\mathrm{CO}_{2}$ and $\mathrm{H}_{2}$ could also provide information on the metabolic condition of the culture and confirm that $\mathrm{H}_{2}$ is produced at a satisfactory rate. Multicomponent gas measurements could also give mechanistic insights into these biological processes, aiding their optimization to maximize $\mathrm{H}_{2}$ yields. Common analytical techniques include gas chromatography (GC) or mass spectrometry (MS); while sensitive and selective, they require expensive equipment and have limitations, including difficulties detecting certain components, long analysis times for GC, and the need for sample preparation, which prevents real-time, in situ monitoring.

Spectroscopic techniques are nonintrusive and provide data in real time for in situ monitoring with high selectivity and sensitivity, including the distinction of isotopomers. ${ }^{11-25}$ Direct absorption techniques, like FTIR spectroscopy, are widely used but are unable to detect molecules such as $\mathrm{H}_{2}, \mathrm{O}_{2}$, or $\mathrm{N}_{2}$. Due to different selection rules, Raman spectroscopy can monitor all relevant components. ${ }^{16-25}$ Despite this, Raman scattering has not found widespread use in trace gas analysis due to its inherent weakness. Trace gas Raman spectroscopy at ambient pressures typically requires the use of large, high power laser systems or sophisticated equipment, which makes it difficult to use as analytical methods. Methods to increase sensitivity include stimulated Raman techniques such as PARS (Photoacoustic Stimulated Raman Spectroscopy) and CARS (Coher-

Received: December 11, 2016

Accepted: January 10, 2017

Published: January 10, 2017 


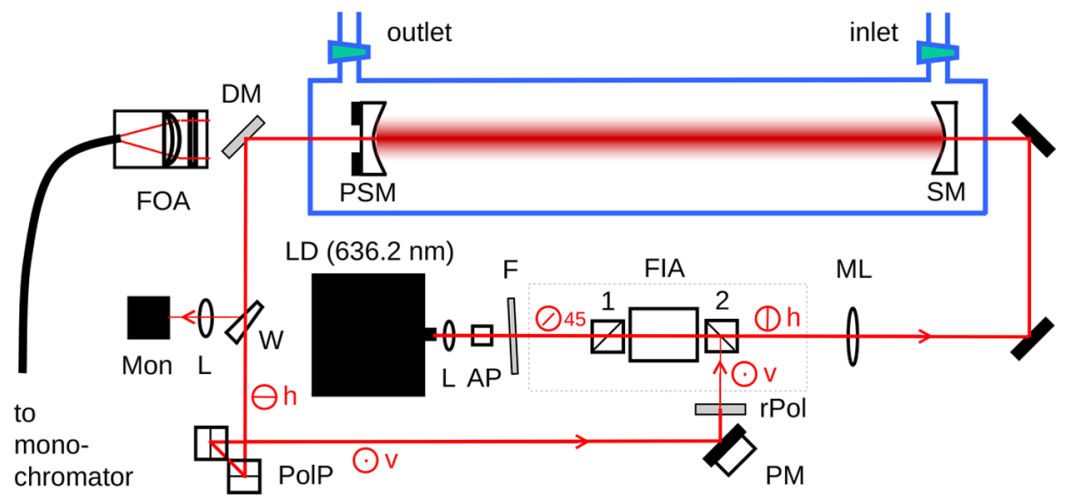

Figure 1. Scheme of the experimental setup (see main text for details).

ent Anti-Stokes Raman Spectroscopy), as well as Fiberenhanced or cavity-enhanced Raman spectroscopy. ${ }^{18-25}$

Recently, we introduced cavity-enhanced Raman spectroscopy with optical feedback diode lasers (CERS), where an inexpensive diode laser is coupled into a high-finesse optical cavity, leading to power enhancement of about 3 orders of magnitude. ${ }^{22,23}$ CERS has high spectral resolution due to the narrow laser line width obtained by controlled optical feedback. With a monochromator of sufficient spectral bandwidth, CERS can collect information on multiple components in a single acquisition. Here we describe the first application of CERS to the analysis of biohydrogen production from pure cultures. To demonstrate the utility of CERS for biohydrogen detection, we chose $\mathrm{H}_{2}$-producing Escherichia coli $(E$. coli) as this model organism is well understood from a genetic and biochemical viewpoint, is easy to grow, and is reasonably amenable to genetic modification needed to improve $\mathrm{H}_{2}$ yields. ${ }^{26-28}$

In the first section, the experimental apparatus and operating principles of CERS are outlined, and advancements made on this technique are described. We then report the application of CERS to the in situ headspace analysis of anaerobic batch cultures of $E$. coli supplemented with D-glucose or glycerol. We show how the kinetics of hydrogen uptake and formation reactions can be followed simultaneously by isotopically labeling the headspace above the culture. Finally, we demonstrate the ability of CERS to identify CO in the gas feed, a potent inhibitor for both $\mathrm{H}_{2}$ producing hydrogenases and many proposed $\mathrm{H}_{2}$ fuel cell technologies, and its effects on hydrogenase activity in whole $E$. coli cells.

\section{EXPERIMENTAL SECTION}

The principle of CERS with optical feedback has been described before, ${ }^{22,23}$ but the current set up contains important improvements. Briefly, $10 \mathrm{~mW}$ laser radiation from a cw-laser diode LD at $636.18 \mathrm{~nm}$ (Hitachi HL6322G) is coupled via lens $\mathbf{L}$, anamorphic prism pair $\mathbf{A P}$, short-pass filter $\mathbf{F}$, and mode matching lens ML into an optical cavity composed of two highly reflective mirrors (Newport SuperMirrors, $R>99.99 \%$ ) SM and PSM (Figure 1). Unwanted back reflections into the laser are prevented by a Faraday rotator isolator assembly, FIA. In previous implementations, two Faraday isolators were used in series to provide good isolation. In the meantime, we have found that one isolator is sufficient if it is carefully tuned for optimal isolation. If the laser wavelength matches the cavity length, an optical resonance builds up laser power inside the cavity by up to 3 orders of magnitude, which greatly increases Raman signals. After the cavity, a dichroic mirror DM separates excitation light from Raman signals, which are coupled into a fiber and transferred to the monochromator (Shamrock SR750-A, with Andor iVac DR32400 camera at $-60{ }^{\circ} \mathrm{C}$ ). Part of the laser light is diverted back to the diode for optical feedback via the polarizing beam splitting cube 2 of FIA, locking the laser to the cavity; the intensity of the fed-back light can be adjusted via a rotating polarizer, rPol. The diode laser itself is linearly polarized at an angle of $+45^{\circ}$ to the optical bench. Polarizer 1 of FIA lets this component pass. The Faraday rotator rotates the polarization plane by $-45^{\circ}$, so that afterward, the light is horizontally polarized with respect to the bench $\left(0^{\circ}\right)$ and passes polarizer 2. The light exiting the optical cavity will also be mainly horizontally polarized, but this would make it unsuitable for optical feedback because, in the return path, polarizer 2 of FIA will only reflect vertically polarized light back to the diode. It is therefore necessary to rotate the polarization plane. This can be achieved by two mirrors or prisms (PolP in Figure 1), which first divert the beam by $90^{\circ}$ up vertically from the bench and then immediately by $90^{\circ}$ horizontally to the right of Figure 1 , changing horizontal into vertical polarization. The light can then enter the Faraday rotator via polarizing beam splitting cube 2 , where it will be optically rotated by $-45^{\circ}$ to become $+45^{\circ}$, which can pass polarizer 1 to feed back into the diode. PolP is essential if the set up uses one Faraday rotator.

The diode injection current is modulated around one cavity mode; in each cycle, the wavelength changes until it is locked to a longitudinal mode of the cavity by optical feedback. Previously, electronic locking circuits and mirrors mounted on piezoelectric transducers (PSM and PM in Figure 1) were used for mode and phase matching. ${ }^{22,23}$ In a significant simplification, we have found that with sufficiently strong optical feedback, the laser will effectively self-lock and electronic mode tracking is not essential. Although resonances are less regular, Raman intensity fluctuations can be very effectively normalized using the $\mathrm{N}_{2}$ Raman peak as an internal standard, if $\mathrm{N}_{2}$ remains constant in the system. At $30 \mathrm{~s}$ acquisition time, noise-equivalent detection limits are about 0.14 mbar $\mathrm{H}_{2}$ using a high-resolution grating $\left(0.8 \mathrm{~cm}^{-1}\right.$ resolution, $500 \mathrm{~cm}^{-1}$ spectral range), ${ }^{22,23}$ and $1 \mathrm{mbar}_{2}$ with a low-resolution grating $\left(12 \mathrm{~cm}^{-1}\right.$ resolution, $4000 \mathrm{~cm}^{-1}$ spectral range). Detection limits, sensitivities, and relative intensities are discussed in detail in our previous publications; $^{22,23}$ for convenience, we include a summary in the Supporting Information (Table S1). Typical Raman spectra with the low resolution grating are shown in Figure 2 (see further below for details of this experiment). Raman intensity is converted to partial pressure using tabulated integrated areas 


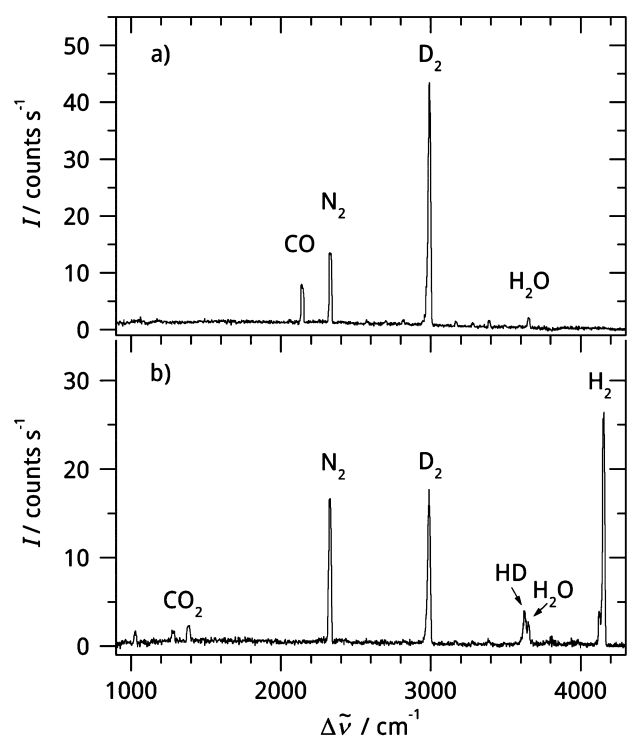

Figure 2. Typical CERS Raman spectra of the culture headspace in the anaerobic fermentation of $98 \mathrm{mM}$ glycerol under an $\mathrm{N}_{2} / \mathrm{D}_{2} / \mathrm{CO}$ atmosphere, (a) observed in the first phase after 76 min with $\mathrm{CO}, \mathrm{N}_{2}$, and $\mathrm{D}_{2}$ present; (b) observed at the end of the second phase, where the $\mathrm{CO}$ was removed.

(Table S1). ${ }^{23}$ At equilibrium, the molarity of a dissolved gas can be calculated from its partial pressure using Henry's law. ${ }^{29}$ A small proportion of dissolved $\mathrm{CO}_{2}$ will react with water to form carbonic acid, which will be at equilibrium with bicarbonate and carbonate ions, depending on the $\mathrm{pH}$. With a typical acidic $\mathrm{pH}$ below 5 at the end of a fermentation experiment, less than $1 \%$ of dissolved $\mathrm{CO}_{2}$ will be lost to carbonic acid and carbonates. The optical cavity is inside a vacuum-tight glass enclosure. Gas inlet and outlet taps allow controlled filling with gas mixtures. To characterize hydrogen leaking, CERS measurements of 1 bar mixtures of $\mathrm{H}_{2} / \mathrm{D}_{2} / \mathrm{N}_{2}$ gave a loss rate of $\mathrm{H}_{2}$ and $\mathrm{D}_{2}$, with a half time of about 22-26 days, with $\mathrm{H}_{2}$ on the lower end of this range and $\mathrm{D}_{2}$ on the higher end.

E. coli (strain K-12 MG1655) was transferred from glycerol stock (maintained at $-80{ }^{\circ} \mathrm{C}$ ) and streaked on sterile LB-agar plates (LB, lysogeny broth, a nutrient rich growth medium). Plates were left overnight at $37^{\circ} \mathrm{C}$ to allow distinct colonies to grow. For each measurement, $50 \mathrm{~mL}$ of sterile $\mathrm{LB}$ was inoculated with a single colony and grown anaerobically in a sealed $50 \mathrm{~mL}$ centrifuge tube for $16 \mathrm{~h}\left(37^{\circ} \mathrm{C}, 200 \mathrm{rpm}\right)$. The culture was added to $200 \mathrm{~mL}$ of fresh, sterile $\mathrm{LB}\left(\mathrm{OD}_{600} \approx 0.2\right.$, optical density at $600 \mathrm{~nm}$ in a $1 \mathrm{~cm}$ cuvette), supplemented with either D-glucose or glycerol and transferred to the CERS apparatus. Bacterial suspensions were kept in the dark with constant stirring at $37^{\circ} \mathrm{C}$ in a $500 \mathrm{~mL}$ round-bottom flask in a thermostated water bath. The flask was connected to the CERS enclosure with short gas transfer tubes, giving a total gas volume of $1330 \mathrm{~mL}$. The transfer tubes and enclosure were kept at about $45{ }^{\circ} \mathrm{C}$ by a thermostated water jacket to avoid condensation. To enhance gas flow, a peristaltic pump $(7 \mathrm{l} / \mathrm{h})$ was used to cycle the flask headspace through the CERS vessel. In a test to characterize the experimental time resolution, $\mathrm{CO}_{2}$ was generated from dry ice added to the flask normally used for biological measurements. The appearance time of $\mathrm{CO}_{2}$ Raman signals in the CERS cell has a half time of about $2.5 \mathrm{~min}$. At the beginning of an experiment, the system was repeatedly evacuated and then flushed with $\mathrm{N}_{2}$ to remove $\mathrm{O}_{2}$ before being filled with $\mathrm{N}_{2}, \mathrm{~N}_{2} / \mathrm{D}_{2}$, or $\mathrm{N}_{2} / \mathrm{D}_{2} / \mathrm{CO}$ gas mixtures to a total pressure of 1 bar. During fermentation, $\mathrm{CO}_{2}$ and $\mathrm{H}_{2}$ were generated, increasing the pressure. At the end of a CERS measurement, the culture was removed from the system. The increase in cell density was characterized by $\mathrm{OD}_{600} \approx 3.5$ (sample $5 \times$ diluted in fresh, sterile LB). Further portions of culture were removed and centrifuged (Sigma 4K15, RCF 5650 $\mathrm{g}$, typically for 20 to $30 \mathrm{~min}$ ). The resulting supernatant was then passed through a $0.22 \mu \mathrm{m}$ filter to remove any residual cellular material and the $\mathrm{pH}$ was measured (Thermo Orion 410 $\mathrm{pH}$ meter), giving a typical $\mathrm{pH} \approx 4.3-4.8$ due to organic acids generated during fermentation. For comparison, fresh LB has $\mathrm{pH} \approx 6.8$. At the beginning of the experiment, the cellular material within the $250 \mathrm{~mL}$ suspension has a typical dry weight of $8 \mathrm{mg}$, which by the end of a typical experiment increased to $60 \mathrm{mg}$, reflecting bacterial growth.

\section{RESULTS AND DISCUSSION}

$\mathrm{H}_{2}$ Production from Anaerobic Batch Cultures with DGlucose. E. coli is able to express four distinct hydrogenases, all of the $[\mathrm{NiFe}]$ type and associated with the inner, cytoplasmic membrane of the cell. ${ }^{28} \mathrm{Hyd}-1$ and Hyd-2 primarily function as uptake hydrogenases. ${ }^{30} \mathrm{Hyd}-3$ is the main $\mathrm{H}_{2}$ producing hydrogenase. In vivo, it forms part of the membrane-anchored formate hydrogenlyase (FHL) complex, which catalyzes the oxidation of formate to $\mathrm{CO}_{2}$ and passes the generated reducing equivalents to the $[\mathrm{NiFe}]$ active site where proton reduction occurs. ${ }^{31}$ Relatively little is known about the fourth hydrogenase Hyd-4, and its physiological role (if any) remains uncertain. ${ }^{32}$ For E. coli and many other facultative anaerobes, $\mathrm{H}_{2}$ production is a strictly fermentative process. Expression of all four hydrogenases is strongly repressed by $\mathrm{O}_{2}$, and the enzymes themselves, with the exception of Hyd-1, are also highly sensitive to even traces of $\mathrm{O}_{2}$. We followed the aerobic metabolism of $E$. coli growing on rich LB medium supplemented with D-glucose. As expected, the $\mathrm{O}_{2}$ pressure decreased, while $\mathrm{CO}_{2}$ increased, but no $\mathrm{H}_{2}$ production was observed, even when $\mathrm{O}_{2}$ was exhausted. Clearly, ensuring the system is $\mathrm{O}_{2}$ free would be critical in large-scale fermentative biohydrogen production. In the absence of $\mathrm{O}_{2}$ or other suitable external electron acceptors such as nitrate, E. coli switches to mixed acid fermentation to derive energy from organic substrates. A mixture of partially oxidized products, $\mathrm{CO}_{2}$ and $\mathrm{H}_{2}$ are generated, the exact distribution governed by the carbon source and the intra- and extracellular environment. ${ }^{33,34}$ During glucose fermentation, the majority of both $\mathrm{CO}_{2}$ and $\mathrm{H}_{2}$ released is generated from oxidation of formate by the FHL complex. To investigate $\mathrm{H}_{2}$ production, we prepared E. coli LB broth cultures supplemented with D-glucose $(40$ or $100 \mathrm{mM})$ and purged with $\mathrm{N}_{2}$ to remove $\mathrm{O}_{2}$. CERS has the advantage of being sensitive to $\mathrm{O}_{2}$, enabling us to check the headspace to ensure its absence and continue to purge if traces are still observed. The composition of the gas phase was then measured for up to 5 days by CERS in order to follow the evolution of volatile components. While the short peptides found in LB can be utilized as a sole carbon and nitrogen source for growth, there was no observable $\mathrm{H}_{2}$ production from cultures grown on nonsupplemented LB.

Figure 3 shows as a typical example the partial pressures of $\mathrm{H}_{2}$ and $\mathrm{CO}_{2}$ in the fermentation of $40 \mathrm{mM}$ glucose. The $\mathrm{H}_{2}$ kinetics has at least three different phases. In the first $2 \mathrm{~h}$, the rise is slow and may give the impression of an induction period; a closer look reveals, however, that $\mathrm{H}_{2}$ is produced almost 


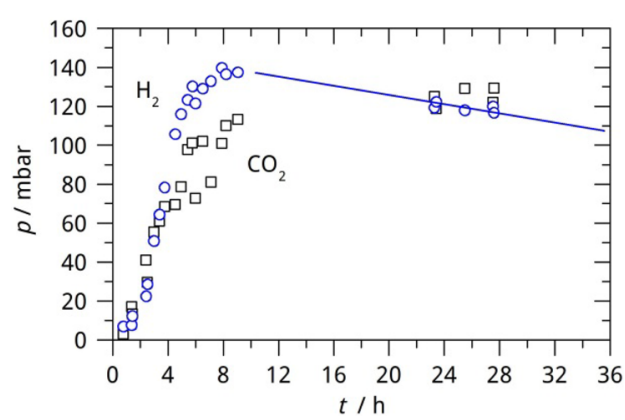

Figure 3. Partial pressures of $\mathrm{CO}_{2}$ (black, squares) and $\mathrm{H}_{2}$ (blue, circles) as a function of time, as observed by CERS in the anaerobic fermentation of $40 \mathrm{mM}$ glucose $(10 \mathrm{mmol})$ by E. coli. At its peak, 140 mbar of $\mathrm{H}_{2}$ is produced, equivalent to $7.1 \mathrm{mmol}$.

immediately, but at a reduced rate. This may reflect differences in $\mathrm{H}_{2}$ metabolism during different stages of growth, perhaps between the lag and exponential phases. The slow phase is followed by a phase of rapid production peaking around $7 \mathrm{~h}$ with a rise half time $t_{1 / 2}$ of about $1 \mathrm{~h}$. At its peak, $140 \mathrm{mbar}$ of $\mathrm{H}_{2}$ is produced, equivalent to $7.1 \mathrm{mmol}$, taking both the solution and headspace into account. With $10 \mathrm{mmol}$ glucose present at the beginning of the experiment, the yield (expressed as $\mathrm{mol} \mathrm{H}_{2} / \mathrm{mol}$ glucose) is 0.71 . After reaching its peak, the $\mathrm{H}_{2}$ concentration starts to decrease, with an extrapolated half time of about 3-4 days. The $\mathrm{CO}_{2}$ partial pressure mirrors that of $\mathrm{H}_{2}$, peaking at $120 \mathrm{mbar}(6.9 \mathrm{mmol})$, although, unlike $\mathrm{H}_{2}$, no significant decay is apparent. The molar ratio of $\mathrm{CO}_{2} / \mathrm{H}_{2}$ at its peak is almost equimolar, indicating that the vast majority of hydrogen originates from the oxidation of formate. Similar behavior was observed with $100 \mathrm{mM}$ glucose: in a typical experiment, 363 mbar $\mathrm{H}_{2}$ was produced, equivalent to 18.5 $\mathrm{mmol}$, and a yield of 0.74 , very similar to the lower glucose concentration. However, $\mathrm{CO}_{2}$ production was proportionally lower than in the $40 \mathrm{mM}$ experiment, with $\mathrm{CO}_{2}$ peaking around $200 \mathrm{mbar}$, corresponding to $11.5 \mathrm{mmol}$ and a molar ratio of $\mathrm{CO}_{2} / \mathrm{H}_{2}$ of only $62 \%$. This might reflect more reducing conditions in the cellular environment, with Hyd-1 or, more likely, Hyd-2 acting as a secondary $\mathrm{H}_{2}$ producing enzyme in a similar way to cultures grown on glycerol.

For both glucose concentrations, $\mathrm{H}_{2}$ was observed to decay, while $\mathrm{CO}_{2}$ remained essentially constant, showing that the cells also exhibit some $\mathrm{H}_{2}$ uptake. Previous work has shown that deletion of genes encoding uptake hydrogenases can increase the overall yield of $\mathrm{H}_{2} \cdot{ }^{35,36}$ Although $\mathrm{Hyd}-3$ has been reported to operate in reverse, coupling $\mathrm{H}_{2}$ oxidation to $\mathrm{CO}_{2}$ reduction to formate, this behavior is probably not relevant under physiological conditions. ${ }^{37}$ In addition, the absence of any observable $\mathrm{CO}_{2}$ uptake indicates that the $\mathrm{H}_{2}$ uptake is primarily due to the respiratory hydrogenases, Hyd-1 and -2 , which are not directly coupled to formate dehydrogenase. Hyd-1 primarily couples the oxidation of $\mathrm{H}_{2}$ to high redox potential electron acceptors, such as $\mathrm{O}_{2}$, and not to low redox potential acceptors. Since the measurements described here were carried out under strictly fermentative conditions where only low potential electron acceptors such as fumarate are present, it seems more likely that the observed $\mathrm{H}_{2}$ uptake is due to Hyd-2 activity. This is in agreement with previous work that showed that deletion of Hyd-1 had little effect on $\mathrm{H}_{2}$ uptake, and a strain carrying deletions in both Hyd-1 and -2 showed no further reduction in $\mathrm{H}_{2}$ uptake over a strain carrying only a Hyd-2 mutation. ${ }^{38}$
Anaerobic Fermentation of Glycerol by $E$. coli. There is a global oversupply of glycerol due to biodiesel production where transesterification of oils generates glycerol-contaminated aqueous waste. ${ }^{39}$ This waste could be a convenient sustainable substrate for organisms such as E. coli, which can utilize glycerol for fermentation under certain conditions. ${ }^{40-42}$ Its higher degree of reduction could be an advantage compared to sugars; glycerol fermentation typically gives increased yields of more reduced and higher value products for the chemical industry. ${ }^{43}$ To investigate $\mathrm{H}_{2}$ production, we prepared E. coli LB broth cultures supplemented with glycerol ( 80 or $200 \mathrm{mM}$ ) and purged with $\mathrm{N}_{2}$ to remove $\mathrm{O}_{2}$. Figure 4 shows a typical

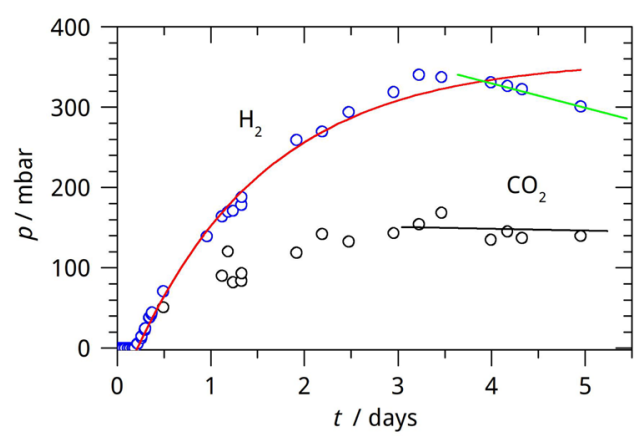

Figure 4. Partial pressures of $\mathrm{CO}_{2}$ and $\mathrm{H}_{2}$ as a function of time as observed by CERS in the anaerobic fermentation of $200 \mathrm{mM}$ glycerol by E. coli.

example of the evolution of $\mathrm{CO}_{2}$ and $\mathrm{H}_{2}$ over 5 days produced by an anaerobic culture supplemented with $200 \mathrm{mM}$ glycerol. The appearance of $\mathrm{H}_{2}$ is approximately described by exponential growth with half time $t_{1 / 2}=23 \mathrm{~h}$ and an apparent delay of about $6 \mathrm{~h}$ (red curve in Figure 4). After reaching its peak at 360 mbar after 3.3 days, the $\mathrm{H}_{2}$ partial pressure shows a slow exponential decay with half time $t_{1 / 2}=6.8 \mathrm{~d}$ (green curve in Figure 4). The $\mathrm{CO}_{2}$ pressure broadly mirrors $\mathrm{H}_{2}$ production, but at 155 mbar, it peaks at a lower value. The lower $\mathrm{CO}_{2} / \mathrm{H}_{2}$ ratio probably reflects the fact that significant amounts of $\mathrm{H}_{2}$ are produced by pathways which do not require simultaneous formation of $\mathrm{CO}_{2}$. This is in agreement with previous work which has shown that Hyd-2 plays also a role in $\mathrm{H}_{2}$ production during glycerol fermentation, where it acts as a "relief valve" to dispose of excess reducing equivalents. ${ }^{44,45}$ For $\mathrm{CO}_{2}$, no distinct decrease is observed after day 3. The observed decrease in $\mathrm{H}_{2}$ thus indicates $\mathrm{H}_{2}$ uptake activity.

Distinctly different behavior is observed for the kinetics of $\mathrm{H}_{2}$ production depending on the carbon source and its concentration. With $40 \mathrm{mM}$ D-glucose, it has a half time of 1 $\mathrm{h}$, tripling to $3 \mathrm{~h}$ for $100 \mathrm{mM}$, whereas $\mathrm{H}_{2}$ production is much slower in glycerol, with a half time of $8 \mathrm{~h}$ for $98 \mathrm{mM}$ glycerol, increasing to $23 \mathrm{~h}$ for $200 \mathrm{mM}$. For D-glucose, the theoretical maximum fermentation yield ( $\mathrm{mol} \mathrm{H}_{2}$ per mol D-glucose) is 2, since up to two formate molecules can be generated from each molecule of glucose via glycolysis and pyruvate cleavage by pyruvate formate-lyase (PFL). ${ }^{33}$ For glycerol, the corresponding maximum yield is 1 . The observed yields of $0.67-0.74$ for D-glucose and $0.27-0.37$ for glycerol are within $27-37 \%$ of the theoretical maximum yield, remarkably independent of the feed stock or its concentration. The observed yield is only a lower limit which could be improved by extraction of $\mathrm{H}_{2}$ when formed, thus preventing accumulation and uptake of $\mathrm{H}_{2}$. Previous work has shown that allowing $\mathrm{H}_{2}$ build up above 
glycerol supplemented cultures is detrimental to growth, which would suggest that constantly siphoning off the produced $\mathrm{H}_{2}$ could be critical for efficient biohydrogen production. ${ }^{46}$ The yields are also lower than those obtained from $\mathrm{H}_{2}$ overproducing mutant strains, which lack uptake hydrogenases and overexpress FHL. ${ }^{35,36}$ This reflects the importance of "rewiring" the mixed acid fermentation pathways in order to maximize carbon flow to formate and minimize losses to undesired products such as lactate or succinate. $\mathrm{H}_{2}$ production is known to be product inhibited; since the experiment was in a sealed system, the buildup of $\mathrm{H}_{2}$ may have contributed to a reduction in the yield. In addition, Hyd-1 and Hyd- 2 primarily operate as $\mathrm{H}_{2}$ oxidizing enzymes, and they may have contributed to removal of $\mathrm{H}_{2}$ in the headspace.

Anaerobic Fermentation under a $D_{2} / N_{2}$ Atmosphere. To separate hydrogen generation and consumption, isotopic labeling with deuterium can be used. $\mathrm{D}_{2}$ labeling of the headspace has previously been employed in combination with membrane inlet mass spectrometry to investigate hydrogenase activity. ${ }^{47}$ One disadvantage with this technique is that gas must be constantly sampled from the headspace, limiting the time for which a labeling experiment could be run and also requiring a correction for the depletion of gas in the headspace. Raman spectroscopy has isotopomer selectivity but does not consume any gas. For isotopic labeling of the headspace, we introduced a large excess of $D_{2}$ at the beginning of the measurement. Batch cultures of E. coli were prepared as before and purged several times to remove any dissolved $\mathrm{O}_{2}$. A defined mixture of $\mathrm{N}_{2} / \mathrm{D}_{2}$ was then introduced into the system to a total pressure of 1 bar (typically 600 mbar $\mathrm{D}_{2}, 400$ mbar $\mathrm{N}_{2}$ ).

Figure 5 shows a typical experiment. Although excess hydrogen is known to inhibit certain classes of hydrogenase,

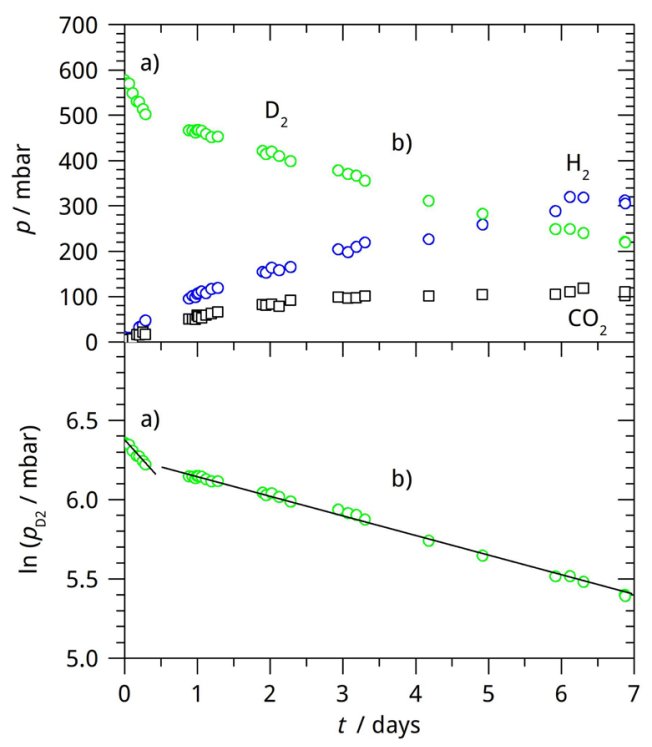

Figure 5. Partial pressures of $\mathrm{H}_{2}, \mathrm{D}_{2}$, and $\mathrm{CO}_{2}$ in the anaerobic fermentation of $40 \mathrm{mM}$ glucose under $\mathrm{D}_{2} / \mathrm{N}_{2}$. The lower plot displays the decay of $\mathrm{D}_{2}$ on a logarithmic scale, showing two distinct kinetic regimes.

there is no delay in the appearance or reduction in the rate of $\mathrm{H}_{2}$ formation. $\mathrm{D}_{2}$ consumption has no lag, indicating that the hydrogenases involved in $\mathrm{D}_{2}$ consumption are already present at the beginning of the measurement. With $40 \mathrm{mM}$ glucose (10 $\mathrm{mmol}$ ), there are two distinct phases of $\mathrm{D}_{2}$ consumption which both adopt pseudo first-order behavior; in phase (a), between 0 to 0.5 days, $\mathrm{D}_{2}$ decays with $t_{1 / 2}=1.4 \mathrm{~d}$ followed by a second phase (b) of slower decay with $t_{1 / 2}=5.0-5.5 \mathrm{~d}$, which continues up to the end of the measurement (0.5-7 days). No distinct transition in $\mathrm{H}_{2}$ or $\mathrm{CO}_{2}$ production is observed between phases (a) and (b). The profiles of $\mathrm{H}_{2}$ and $\mathrm{CO}_{2}$ are distinctly different: $\mathrm{CO}_{2}$ rises to its peak value of about 100 mbar $(5.8 \mathrm{mmol})$ at $3 \mathrm{~d}$, then it remains essentially constant. $\mathrm{H}_{2}$, however, increases for a longer time, reaching a plateau of 340 mbar $(17.3 \mathrm{mmol})$ after $6-7$ days. In the $40 \mathrm{mM}$ glucose experiments with and without $\mathrm{D}_{2}$, approximately the same amount of $\mathrm{CO}_{2}$ is produced; it thus seems reasonable to assume that a similar amount of formate is oxidized by the FHL complex, corresponding to around $7.1 \mathrm{mmol} \mathrm{H}_{2}$. After $7 \mathrm{~d}$, about 350 mbar $(17.9 \mathrm{mmol})$ of $\mathrm{D}_{2}$ is consumed and an additional $10 \mathrm{mmol}$ more $\mathrm{H}_{2}$ is produced than would be expected from fermentation alone. This excess can be accounted for if $56 \%$ of the $\mathrm{D}_{2}$ consumed is converted to $\mathrm{H}_{2}$ through isotope exchange with the solvent. This suggests that some of the consumed $\mathrm{D}_{2}$ is coupled either directly (through $\mathrm{H} / \mathrm{D}$ exchange at a hydrogenase active site) or indirectly (perhaps via intermediate electron donation back into the quinone pool) to proton reduction. Such $\mathrm{D} / \mathrm{H}$ isotope exchange has been well reported in the literature. ${ }^{47}$ Rather unusually for such labeling experiments, there is no significant formation of the mixed isotopomer $\mathrm{HD}$; final $\mathrm{HD}$ pressures are typically below 15 mbar. In contrast, in previously reported experiments, levels of $\mathrm{HD}$ comparable to the added $\mathrm{D}_{2}$ were observed using isolated hydrogenases, membranes, or cell extracts from a variety of organisms. ${ }^{47}$ A similar absence of $\mathrm{HD}$ was, however, observed for purified hydrogenases obtained from Azotobacter vinelandii and Ralstonia eutropha (now Cupriavidus necator) when incubated under $\mathrm{D}_{2}$ in protonated buffer. $^{48,49}$

To probe $\mathrm{H}_{2}$ uptake activity during glycerol fermentation, experiments were performed under an $\mathrm{N}_{2} / \mathrm{D}_{2}$ atmosphere (typically 600 mbar $\mathrm{D}_{2}, 400$ mbar $\mathrm{N}_{2}, 98 \mathrm{mM}$ glycerol (25 $\mathrm{mmol}), 3$ repeats). Figure 6 shows a typical experiment. Samples consistently show a single phase (labeled " $b$ " in Figure 6), up to day 7 , characterized by an exponential decay with $t_{1 / 2}$ $=5.0-5.7 \mathrm{~d}$, very similar to the phase (b) in glucosesupplemented samples. By day 7, typically around $350 \mathrm{mbar}$ (17.9 mmol) of $\mathrm{D}_{2}$ is consumed. $\mathrm{H}_{2}$ continues to rise and appears to start to plateau at a partial pressure of $480 \mathrm{mbar}$ (24.5 mmol) around day 6-7. Unlike glucose fermentation under $\mathrm{D}_{2}, \mathrm{CO}_{2}$ production does not stop early, but continues to increase, with the profile closely mirroring that of $\mathrm{H}_{2}$. A similar plateau is observed in $\mathrm{CO}_{2}$ around day 6-7 with $200 \mathrm{mbar}$ (11.5 mmol) produced, which far exceeds the amount of $\mathrm{CO}_{2}$ produced in glycerol samples without $\mathrm{D}_{2}$. As with glucose, no significant formation of $\mathrm{HD}$ is observed. Assuming a similar $\mathrm{H}_{2}$ fermentation yield as in the experiments without $\mathrm{D}_{2}$, the excess of $\mathrm{H}_{2}$ produced in phase (b) is of the order of $17-19 \mathrm{mmol}$; to account for this by $\mathrm{D}_{2}$ conversion, almost the entire $\mathrm{D}_{2}$ consumed would have to be converted to $\mathrm{H}_{2}$, a much higher percentage than in the case of glucose. The assumption of similar fermentation yields would also be at variance with the higher amount of $\mathrm{CO}_{2}$ produced. The observations that $\mathrm{CO}_{2}$ production does not stop early but continues rising with $\mathrm{H}_{2}$, and that more $\mathrm{CO}_{2}$ is produced indicates a higher fermentation yield of $\mathrm{H}_{2}$ from glycerol in the presence of $\mathrm{D}_{2}$, contrary to the behavior in glucose. A significant amount of the excess $\mathrm{H}_{2}$ is then expected to be due to the increased fermentation, and the 


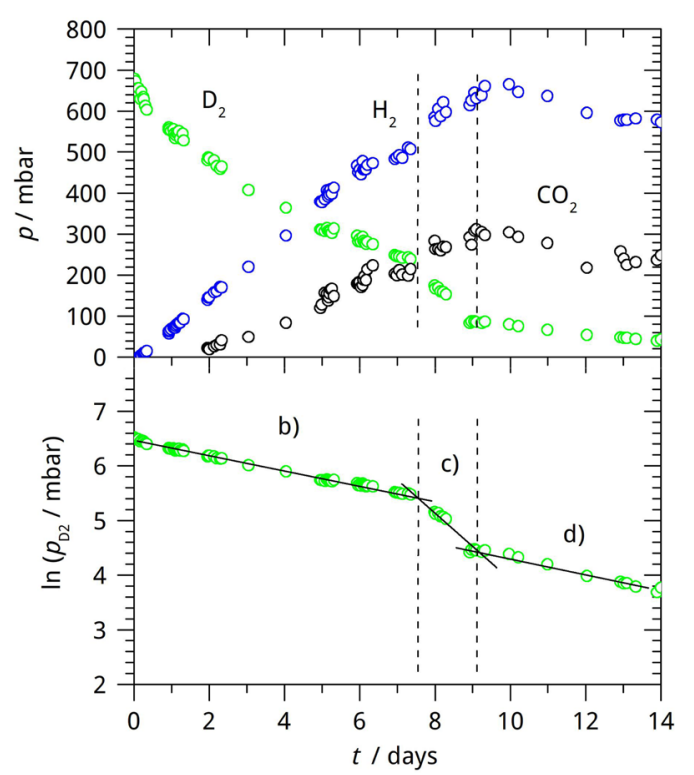

Figure 6. Partial pressures of $\mathrm{H}_{2}, \mathrm{D}_{2}$, and $\mathrm{CO}_{2}$ in the anaerobic fermentation of $98 \mathrm{mM}$ glycerol under $\mathrm{D}_{2} / \mathrm{N}_{2}$. The lower plot displays the decay of $\mathrm{D}_{2}$ on a logarithmic scale, showing three distinct kinetic regimes.

balance from the conversion of $\mathrm{D}_{2}$ is then closer to the $56 \%$ conversion estimated for glucose. A tentative explanation could be that $\mathrm{D}_{2}$ triggers more formate production during mixed acid fermentation which is then split into $\mathrm{H}_{2}$ and $\mathrm{CO}_{2}$. Clearly more work is required to understand the underpinning mechanisms of this increased fermentation yield. If these mechanisms are better understood, conditions in biohydrogen production from glycerol could possibly be optimized to significantly increase the hydrogen yield.

In all three repeat experiments with glycerol, we observed a single event (labeled " $c$ " in Figure 6) at about day 8-10, just after $\mathrm{H}_{2}$ and $\mathrm{CO}_{2}$ appear to plateau and typically lasting for 1 to 2 days. During this event, $\mathrm{D}_{2}$ consumption significantly increases, with $t_{1 / 2}=0.8-1.5 \mathrm{~d}$; afterward, it resumes a slower decay as before (labeled "d" in Figure 6). During event (c), 8.4 mmol of $\mathrm{D}_{2}$ is lost, and $6.6 \mathrm{mmol}$ of $\mathrm{H}_{2}$ and $5.5 \mathrm{mmol}$ of $\mathrm{CO}_{2}$ are gained. The sudden change is striking, with accelerated $\mathrm{D}_{2}$ consumption occurring with increased $\mathrm{H}_{2}$ and $\mathrm{CO}_{2}$ production, which suggests that this is not simply $\mathrm{D} / \mathrm{H}$ exchange. It may reflect increased FHL activity, perhaps due to a sudden surge of formate into the cytoplasm. The phase of rapid $\mathrm{D}_{2}$ consumption occurs just after $\mathrm{H}_{2}$ and $\mathrm{CO}_{2}$ begin to plateau, which may indicate that it coincides with exhaustion of glycerol or some intermediate metabolite. It could also be related to changes in $\mathrm{pH}$ or redox potential, as both impact hydrogenase expression and activity. For convenience, all results on glucose and glycerol fermentation under $\mathrm{N}_{2}$ and $\mathrm{N}_{2} / \mathrm{D}_{2}$ are summarized in the Supporting Information (SI Tables S2 and S3), including yields and indicating the number of repeat experiments.

Although the precise mechanism of hydrogenase turnover is still debated, a recent high resolution crystallographic study has obtained a structure of a hydride intermediate for a $[\mathrm{NiFe}]$ hydrogenase, confirming that $\mathrm{H}_{2}$ is cleaved and formed heterolytically. ${ }^{50} \mathrm{~A}$ further oxidation step is then required before the hydride can be oxidized and then removed from the active site as a proton. The absence of major HD formation in our measurements could indicate that the second oxidation step is much faster than a recombination of the deuteride intermediate with a solvent-derived proton and release of HD. ${ }^{47}$ Alternatively, HD may be formed but recaptured by the same active site (a cage effect mechanism) or it may simply indicate that, at the enzyme concentrations present in culture, any HD will undergo more encounters before being released to the environment as $\mathrm{H}_{2} \cdot{ }^{51} \mathrm{HD}$ might also have a large kinetic isotope effect favoring uptake over $\mathrm{D}_{2}$, so that it is preferentially consumed once formed. These mechanistic details of isotope conversion can be resolved in future experiments employing the CERS technique.

CO Blocks Anaerobic Hydrogen Production of E. coli with Glycerol. $\mathrm{CO}$ is a potent inhibitor of many metalloenzymes, including certain classes of hydrogenases. Many of the $\mathrm{O}_{2}$ tolerant hydrogenases, such as E. coli Hyd-1, are also typically more resistant to $\mathrm{CO}$ inhibition, whereas $\mathrm{O}_{2}$ sensitive hydrogenases, such as E. coli Hyd-2 and -3, are inhibited by CO. ${ }^{30,51-53}$ To study this effect, we introduced $\mathrm{CO}$ into the headspace along with $\mathrm{N}_{2}$ and $\mathrm{D}_{2}$ during the purge step. After leaving the culture under the same $\mathrm{CO} / \mathrm{D}_{2} / \mathrm{N}_{2}$ atmosphere for a day, the system was purged several times with $\mathrm{N}_{2}$, and an $\mathrm{N}_{2}$ / $\mathrm{D}_{2}$ atmosphere was reintroduced into the system. The headspace was then monitored for a further 9 days (see Figure 2). Observed partial pressures of the different components are shown in Figure 7. The presence of $\mathrm{CO}$ in the headspace

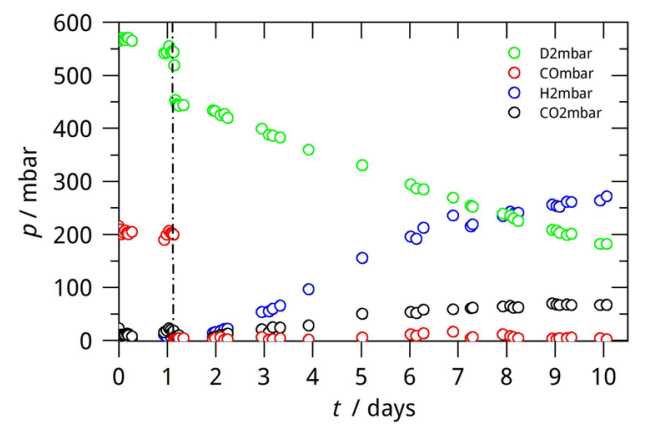

Figure 7. Partial pressures of $\mathrm{H}_{2}, \mathrm{D}_{2}, \mathrm{CO}_{2}$, and $\mathrm{CO}$ in the anaerobic fermentation of $98 \mathrm{mM}$ glycerol. First phase from 0 to $1 \mathrm{~d}$ with $\mathrm{CO}$ present; second phase from 1 to $10 \mathrm{~d}$ where $\mathrm{CO}$ has been removed.

completely inhibited formation of $\mathrm{H}_{2}$ and $\mathrm{CO}_{2}$ and partially inhibited $\mathrm{D}_{2}$ uptake. Since Hyd-1 is the only hydrogenase in $E$. coli known to have some level of $\mathrm{CO}$ tolerance, we propose that the limited $\mathrm{D}_{2}$ uptake activity during the first day must be due to Hyd-1. The half-life of 13.6 days for $\mathrm{D}_{2}$ consumption is considerably longer than in the measurements where $\mathrm{CO}$ was not introduced into the headspace. This supports the hypothesis that either or both of Hyd-2 and Hyd-3, which are strongly inhibited under a $\mathrm{CO}$ atmosphere, are more important than Hyd-1 under these conditions. A partial recovery of $\mathrm{H}_{2}$ producing activity is observed when $\mathrm{CO}$ is removed. Recovery is not instantaneous, with a delay of around 0.5 days before the onset of $\mathrm{D}_{2}$ oxidation and 1 day before $\mathrm{H}_{2}$ production. This may reflect the growth of new cells rather than recovery of cells present during the $\mathrm{CO}$ inhibition phase. As in the previous experiments, $\mathrm{HD}$ is only formed to a minor extent (see Figure 2). To our knowledge, this is the first demonstration of selective $\mathrm{CO}$ inhibition of hydrogenases in $E$. coli whole cells. 


\section{CONCLUSIONS}

Cavity-enhanced Raman spectroscopy (CERS) with optical feedback cw-diode lasers is a sensitive and selective analytical tool for in situ, multicomponent, and isotope selective gas measurements. We have demonstrated the operation with just one Faraday isolator and without active phase and mode matching, greatly simplifying the setup. The improved setup has been employed in its first application to study hydrogen production and consumption by E. coli. Under anaerobic conditions, cultures grown on either D-glucose or glycerol produce $\mathrm{H}_{2}$ and $\mathrm{CO}_{2}$, simultaneously consuming some of the produced $\mathrm{H}_{2}$. By introducing $\mathrm{D}_{2}$, the kinetic processes of hydrogen production and consumption could be separated due to the distinct signatures of each isotopomer. The experiments show that some of the $\mathrm{D}_{2}$ consumed is converted back to $\mathrm{H}_{2}$. $\mathrm{HD}$ is only formed as a minor component. Different phases with distinctly different kinetic regimes of $\mathrm{H}_{2}$ and $\mathrm{CO}_{2}$ production and $\mathrm{D}_{2}$ consumption were identified. The presence of $\mathrm{D}_{2}$ seems to increase the $\mathrm{H}_{2}$ fermentation yield in glycerol. If the mechanisms of this effect are better understood, conditions in biohydrogen production from waste glycerol could be optimized. Although the measurements described here deal with a pure culture, mixed consortia of microorganisms, such as those obtained from biogas slurry, could prove to be a more economical inoculant. ${ }^{54}$ In these systems, heat treatment is required in order to remove methanogens, which consume $\mathrm{H}_{2}$ and generate methane. As previously demonstrated by our group, ${ }^{22,23} \mathrm{CERS}$ is able to distinguish $\mathrm{H}_{2}$ and $\mathrm{CH}_{4}$, so a similar CERS-based approach could be useful for developing and optimizing these systems, confirming the absence of methanogenic organisms by checking the headspace for methane. Due to its unique analytical capabilities, CERS can supplement existing techniques to obtain relevant insights into the biochemistry of the uptake and production of gases and volatile species.

\section{ASSOCIATED CONTENT}

\section{S Supporting Information}

The Supporting Information is available free of charge on the ACS Publications website at DOI: 10.1021/acs.analchem.6b04924.

Table S1, CERS Raman characteristics of compounds measured; Table S2, Yields and kinetics of $\mathrm{H}_{2}$ production during anaerobic fermentation under an $\mathrm{N}_{2}$ atmosphere; Table S3, Observed yield and kinetics of $\mathrm{H}_{2}$ production and $\mathrm{D}_{2}$ consumption during anaerobic fermentation under an $\mathrm{N}_{2} / \mathrm{D}_{2}$ atmosphere (PDF).

\section{AUTHOR INFORMATION}

\section{Corresponding Author}

*E-mail: m.hippler@sheffield.ac.uk.

\section{ORCID ${ }^{\circ}$}

Michael Hippler: 0000-0002-3956-3922

\section{Notes}

The authors declare no competing financial interest.

\section{ACKNOWLEDGMENTS}

We are very grateful to Profs. R. K. Poole and J. Green (University of Sheffield) for substantial help, support, and advice. This work is funded by the University of Sheffield and the NERC (grant NE/I000844/1) and EPSRC (DTA Ph.D. scholarship to T.W.S.) research councils.

\section{REFERENCES}

(1) Hosseini, S. E.; Wahid, M. A. Renewable Sustainable Energy Rev. 2016, 57, 850-866.

(2) Eberle, U.; Felderhoff, M.; Schüth, F. Angew. Chem., Int. Ed. 2009, 48, 6608-6630.

(3) Turner, J.; Sverdrup, G.; Mann, M. K.; Maness, P. C.; Kroposki, B.; Ghirardi, M.; Evans, R. J.; Blake, D. Int. J. Energy Res. 2008, 32, 379-407.

(4) Armaroli, N.; Balzani, V. Angew. Chem., Int. Ed. 2007, 46, 52-66.

(5) Yasin, N. H. M.; Mumtaz, T.; Hassan, M. A.; Rahman, N. A. J. Environ. Manage. 2013, 130, 375-385.

(6) Show, K. Y.; Lee, D. J.; Tay, J. H.; Lin, C. Y.; Chang, J. S. Int. J. Hydrogen Energy 2012, 37, 15616-15631.

(7) Lee, H. S.; Vermaas, W. F. J.; Rittmann, B. E. Trends Biotechnol. 2010, 28, 262-271.

(8) Gupta, S. K.; Kumari, S.; Reddy, K.; Bux, F. Environ. Technol. 2013, 34, 1653-1670.

(9) Vazquez, G. D.; Arriaga, G. S.; Mondragón, F. A.; Rodríguez, A. L.; Colunga, L. M. R.; Flores, E. R. Rev. Environ. Sci. Bio/Technol. 2008, 7, 27-45.

(10) Lubitz, W.; Ogata, H.; Rüdiger, O.; Reijerse, E. Chem. Rev. 2014, 114, 4081-4148.

(11) Quack, M. Annu. Rev. Phys. Chem. 1990, 41, 839-874.

(12) Sigrist, M. W. Air Monitoring by Spectroscopic Techniques; Chemical Analysis Series 127; Wiley: New York, 1994.

(13) Hippler, M.; Mohr, C.; Keen, K.; McNaghten, E. J. Chem. Phys. 2010, 133, 044308 (1-8).

(14) Hippler, M.; Miloglyadov, E.; Quack, M.; Seyfang, G. Mass and Isotope-Selective Infrared Spectroscopy. In Handbook of HighResolution Spectroscopy; Quack, M., Merkt, F., Eds.; John Wiley: Chichester, 2011; pp 1069-1118.

(15) Tinajero-Trejo, M.; Rana, N.; Nagel, C.; Jesse, H. E.; Smith, T. W.; Wareham, L. K.; Hippler, M.; Schatzschneider, U.; Poole, R. K. Antioxid. Redox Signaling 2016, 24 (14), 765-780.

(16) Weber, A. High-resolution Raman Spectroscopy of Gases. In Handbook of High-Resolution Spectroscopy; Quack, M., Merkt, F., Eds.; John Wiley: Chichester, 2011; pp 1153-1236.

(17) Eichmann, S. C.; Kiefer, J.; Benz, J.; Kempf, T.; Leipertz, A.; Seeger, T. Meas. Sci. Technol. 2014, 25, 075503.

(18) Lavorel, B.; Millot, G.; Rotger, M.; Rouillé, G.; Berger, H.; Schrötter, H. W. J. Mol. Struct. 1992, 273, 49-59.

(19) Spencer, C. L.; Watson, V.; Hippler, M. Analyst 2012, 137, 1384-1388.

(20) Hanf, S.; Keiner, R.; Yan, D.; Popp, J.; Frosch, T. Anal. Chem. 2014, 86, 5278-5285.

(21) James, T. M.; Rupp, S.; Telle, H. H. Anal. Methods 2015, 7, $2568-2576$

(22) Salter, R.; Chu, J.; Hippler, M. Analyst 2012, 137, 4669-4676.

(23) Hippler, M. Anal. Chem. 2015, 87, 7803-7809.

(24) Keiner, R.; Frosch, T.; Massad, T.; Trumbore, S.; Popp, J. Analyst 2014, 139, 3879-3884.

(25) Jochum, T.; Michalzik, B.; Bachmann, A.; Popp, J.; Frosch, T. Analyst 2015, 140 (9), 3143-3149.

(26) Colunga, L. M. R; Rodríguez, A. L. Rev. Environ. Sci. Bio/ Technol. 2015, 14, 123-135.

(27) Maeda, T.; Sanchez-Torres, V.; Wood, T. K. Microb. Biotechnol. 2012, 5, 214-225.

(28) Sargent, F. The model [NiFe]-hydrogenases of Escherichia coli. In Advances in Microbiol Physiology; Poole, R. K., Ed.; Elsevier, 2016; Vol. 68 .

(29) www.webbook.nist.gov; accessed 23/3/2016.

(30) Lukey, M. J.; Parkin, A.; Roessler, M. M.; Murphy, B. J.; Harmer, J.; Palmer, T.; Sargent, F.; Armstrong, F. A. J. Biol. Chem. 2010, 285, 3928-3938.

(31) McDowall, J. S.; Hjersing, M. C.; Palmer, T.; Sargent, F. FEBS Lett. 2015, 589, 3141-3147. 
(32) Skibinski, D. A. G.; Golby, P.; Chang, Y. S.; Sargent, F.; Hoffman, R.; Harper, R.; Guest, J. R.; Attwood, M. M.; Berks, B. C.; Andrews, S. C. J. Bacteriol. 2002, 184, 6642-6653.

(33) Bock, A.; Sawers, G. Fermentation. In Escherichia coli and Salmonella, Cellular and Molecular Biology, 2nd ed.; Neidhardt, F. C., Curtiss, R., Ingraham, J. I., Lin, E. C. C., Low, K. B., Magasanik, B., Reznikoff, W., Riley, Schaechter, M., Umbarger, H. E., Eds.; American Society for Microbiology: Washington, D.C., 1996; Chapter 18.

(34) Elsharnouby, O.; Hafez, H.; Nakhla, G.; El Naggar, M. H. Int. J. Hydrogen Energy 2013, 38, 4945-4966.

(35) Maeda, T.; Sanchez-Torres, V.; Wood, T. K. Appl. Microbiol. Biotechnol. 2007, 77, 879-890.

(36) Maeda, T.; Sanchez-Torres, V.; Wood, T. K. Microb. Biotechnol. 2008, 1, 30-39.

(37) Pinske, C.; Sargent, F. MicrobiologyOpen 2016, 5, 721-737.

(38) Redwood, M. D.; Mikheenko, I. P.; Sargent, F.; Macaskie, L. E. FEMS Microbiol. Lett. 2008, 278, 48-55.

(39) Dobson, R.; Gray, V.; Rumbold, K. J. Ind. Microbiol. Biotechnol. 2012, 39, 217-226.

(40) Chaudhary, N.; Ngadi, M. O.; Simpson, B. K.; Kassama, L. S. Adv. Chem. Eng. Sci. 2011, 1, 83-89.

(41) Trchounian, K.; Trchounian, A. Renewable Energy 2015, 83, 345-351.

(42) Dharmadi, Y.; Murarka, A.; Gonzalez, R. Biotechnol. Bioeng. 2006, 94, 821-829.

(43) Clomburg, J. M.; Gonzalez, R. Trends Biotechnol. 2013, 31, 2028.

(44) Trchounian, K.; Soboh, B.; Sawers, R. G.; Trchounian, A. Cell Biochem. Biophys. 2013, 66, 103-108.

(45) Blbulyan, S.; Trchounian, A. Arch. Biochem. Biophys. 2015, 579, $67-72$.

(46) Murarka, A.; Dharmadi, Y.; Yazdani, S. S.; Gonzalez, R. Appl. Environ. Microbiol. 2008, 74, 1124-1135.

(47) Vignais, P. M. Coord. Chem. Rev. 2005, 249, 1677-1690.

(48) McTavish, H.; Sayavedra-Soto, L. A.; Arp, D. J. Biochim. Biophys.

Acta, Protein Struct. Mol. Enzymol. 1996, 1294, 183-190.

(49) Bernhard, M.; Buhrke, T.; Bleijlevens, B.; De Lacey, A. L.; Fernandez, V. M.; Albracht, S. P. J.; Friedrich, B. J. Biol. Chem. 2001, 276, 15592-15597.

(50) Ogata, H.; Nishikawa, K.; Lubitz, W. Nature 2015, 520, 571574.

(51) Tamiya, N.; Miller, S. L. J. Biol. Chem. 1963, 238, 2194-2198.

(52) Vincent, K. A.; Cracknell, J. A.; Lenz, O.; Zebger, I.; Friedrich, B.; Armstrong, F. A. Proc. Natl. Acad. Sci. U. S. A. 2005, 102, 1695116954.

(53) McDowall, J. S.; Murphy, B. J.; Haumann, M.; Palmer, T.; Armstrong, F. A.; Sargent, F. Proc. Natl. Acad. Sci. U. S. A. 2014, 111, E3948-E3956.

(54) Li, C.; Fang, H. H. P. Crit. Rev. Environ. Sci. Technol. 2007, 37, $1-39$. 